rates were observed overall $(\mathrm{HR}=1.37,1.25-1.49)$, among foremen/forewomen $(\mathrm{HR}=1.35,1.04-1.77)$, excavating and grading occupations $(\mathrm{HR}=1.37,1.18-1.58)$, labourers $(\mathrm{HR}=1.55,1.29-1.86)$, and non-specified excavating/grading/ paving occupations $(\mathrm{HR}=1.35,1.15-1.59)$. Non-significant positive rates of bladder cancer were also observed overall $(\mathrm{HR}=1.08,0.93-1.26)$, among excavating and grading workers $(\mathrm{HR}=1.13,0.88-1.45)$, and non-specified excavating/graving/ paving occupations ( $\mathrm{HR}=1.29,1.00-1.68)$.

Conclusion These results identify construction groups with high cancer risk, including excavating and grading occupations, potentially due to DEE exposure, though co-exposure to other carcinogens (e.g. silica) is possible. Targeted prevention resources could reduce exposure and subsequently occupational cancer risk, but would benefit from more detailed DEE exposure information.

\section{$0-32$ THE VALUE OF PLAIN CHEST RADIOGRAPH AS A DIAGNOSTIC TOOL FOR TB RELATIVE TO GENEXPERT AMONG EX-GOLD MINERS IN LESOTHO}

'Botembetume Maboso, Rodney Ehrlich. 'University of Cape Town, South Africa

\subsection{6/OEM-2021-EPI.39}

Background The World Health Organisation and many national guidelines for TB management recommend treatment initiation in the presence of symptoms such as cough, weight loss, night sweats and or fever, and radiological changes suggestive of $\mathrm{TB}$ with or without bacteriological confirmation. However, none of the studies that investigated the value of plain chest radiograph (CXR) has been done in the Southern African ex-gold miner population. Given the characteristics of this population - a high prevalence of silicosis, past TB and recurrent $\mathrm{TB}$ and post-TB lung destruction -application of the above recommendation may lead to unnecessary TB treatment. Objectives To assess the performance of the screening CXR in the diagnosis of active TB disease among former gold miners from the South African mines using GeneXpert as the reference standard.

Methods We analysed the medical history information, CXR, and GeneXpert test results in a group of ex-miners examined between 2017 and 2018 at Mafeteng Occupational Health Service Centre (OHSC), Lesotho. We excluded those on TB treatment and those within twelve months of TB-treatment completion at the time of the visit. CXRs were read by a medical doctor with training and experience in the reading of pneumoconiosis and TB. A set of 300 of the CXRs were cross-read by two occupational medicine specialists with mining medicine experience, with $80-90 \%$ agreement with clinic readings.

Results We analysed information on 2572 subjects. The prevalence of active TB on GeneXpert was 3\%, with CXR sensitivity 0.97 , specificity 0.41 , positive predictive value 0.05 , and negative predictive value 0.99 .

Conclusion A CXR negative for TB is a valuable tool in ruling out active TB disease among ex-gold miners. However, its poor predictive value, given the high background prevalence of silicosis and previous TB in this population, makes bacteriological confirmation necessary before initiating $\mathrm{TB}$ treatment in ex-gold miners.

\section{$0-72$ HOW TO PREDICT OBSTRUCTIVE SLEEP APNOEA IN PROFESSIONAL DRIVERS?}

${ }^{1}$ Elisabeta Pletea, Nicole Majery, Camelia Trandafir. 'STM (Senvice de Santé au Travail Multisectoriel), Luxembourg, Belgium

\subsection{6/OEM-2021-EPI.40}

Objective Developing better tools to screen obstructive sleep apnea (OSA) in professional drivers is fundamental in occupational health services, given the serious consequences this condition can have on their safety.

Methods 1928 professional drivers were screened for OSA for three years using Berlin and Epworth questionnaires. $10.3 \%$ were suspected to be positive for OSA and send for polysomnography tests. We had a return of 39 data. We analysed the results with the MAP index because it has shown a high sensitivity and specificity in studies on professional drivers. The MAP index is easy to use and combines selfreported apnea symptoms (snoring and cessation of breathing) as well as objective data like age, sex, and body mass index (BMI). As the apnoea symptoms regarding snoring and observed apnoea are often not properly reported, we included the questions of the Berlin questionnaire about tiredness in order to improve the predictability and calculated a MAP2. To determine diagnostic test accuracy, we calculated both MAP indexes with apnea-hypopnea index (AHI) criteria already published in articles. Sensitivity, specificity, negative and positive predictive values (NPV, PPV) and area under curve (AUC) for receiver operating characteristic (ROC) were analysed. Predictive utility of both indexes was examined by characteristic variables, age $(<50$ or $\geq 50$ years) and BMI $\left(<30\right.$ or $\left.\geq 30 \mathrm{~kg} / \mathrm{m}^{2}\right)$.

Results Employing the MAP_index1 for OSA, the sensitivity obtained was $63.3 \%$ and specificity was $41.2 \%$. The PPV was $58.3 \%$ and NPV was $46.7 \%$. The AUC was 0.524 (95\% CI $0.339-0.709)$. The accuracy was higher in younger versus older drivers (AUC 0.701 versus 0.620). Sensitivity for Map index 2 was $59.1 \%$ and specificity was $52.9 \%$. The PPV was $61.9 \%$ and the NPV was $50.0 \%$. The AUC was $0.560(95 \%$ CI $0.376-0.744)$.

Conclusions The MAP 2 was had lower sensitivity but higher specificity. It cannot be considered as a better tool to predict obstructive sleep apnoea.

\section{0-254 TEMPORAL TREND AND SPATIAL PATTERN OF MORTALITY FOR MALIGNANT PLEURAL MESOTHELIOMA IN TAIWAN DURING 1975-2019}

${ }^{1}$ Jing-Yi Wu, Tsung-Hsueh Lu, Lukas Jyuhn-Hsiarn Lee. ${ }^{1}$ National Cheng Kung University, Taiwan

\subsection{6/OEM-2021-EPI.41}

Introduction Malignant pleural mesothelioma (MPM) is a rare but aggressive malignancy associated with asbestos exposure for more than $80 \%$ of cases. The average latency of MPM is twenty to forty years. The history of asbestos use in Taiwan showed the massive importation during 1960-1980, but was under control by legislation since 1989, and was totally banned by legislation since 2018 . 